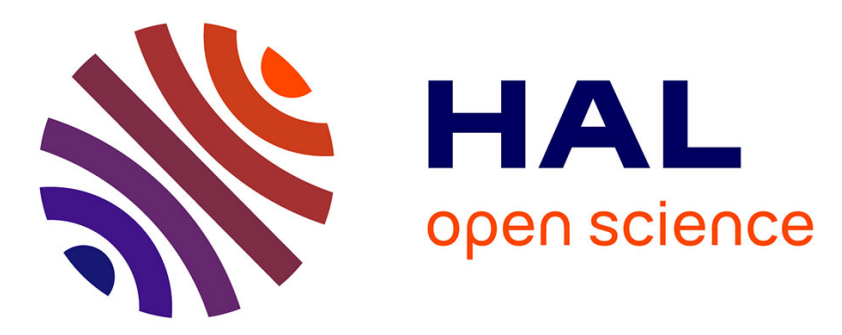

\title{
A mixed variational formulation of dynamic viscoelastic problems with adhesion and friction
}

Marius Cocou

\section{To cite this version:}

Marius Cocou. A mixed variational formulation of dynamic viscoelastic problems with adhesion and friction. Mechanics Research Communications, 2020, pp.103642. 10.1016/j.mechrescom.2020.103642 . hal-03085400

\author{
HAL Id: hal-03085400 \\ https://hal.science/hal-03085400
}

Submitted on 21 Dec 2020

HAL is a multi-disciplinary open access archive for the deposit and dissemination of scientific research documents, whether they are published or not. The documents may come from teaching and research institutions in France or abroad, or from public or private research centers.
L'archive ouverte pluridisciplinaire HAL, est destinée au dépôt et à la diffusion de documents scientifiques de niveau recherche, publiés ou non, émanant des établissements d'enseignement et de recherche français ou étrangers, des laboratoires publics ou privés. 


\title{
A mixed variational formulation of dynamic viscoelastic problems with adhesion and friction
}

\author{
Marius Cocou 1 \\ Aix Marseille Univ, CNRS, Centrale Marseille, LMA UMR 7031, Marseille, \\ France
}

Dedicated to the memory of Professor Nicolaie Dan Cristescu

\section{Keywords}

Dynamic viscoelastic problems, pointwise contact conditions, adhesion, slip depending friction, mixed variational formulation.

\begin{abstract}
The aim of this work is to study a class of dynamic contact problems coupling adhesion and friction between two viscoelastic bodies with nonlinear viscosity operators. The boundary conditions concern relaxed unilateral contact, pointwise friction and adhesion including possible recoverable behavior. A mixed variational formulation of these problems is given as a five-field evolution implicit equation coupled with a differential inclusion describing the evolution of the intensity of adhesion. Based on several estimates and a classical fixed point theorem for multivalued functions, the existence of a strong variational solution is proved.
\end{abstract}

\section{Introduction}

This paper deals with the analysis of a class of nonsmooth dynamic contact problems which describe various surface interactions between two viscoelastic bodies with nonlinear viscosity operators. These interactions include some relaxed unilateral contact, friction with slip depending coefficient of friction, and complex adhesion conditions.

The quasistatic elastic problems with unilateral contact and local Coulomb friction have been studied in [1, 32, 33] and extensions by taking into account

\footnotetext{
${ }^{1}$ Corresponding author:

Marius Cocou, Laboratoire de Mécanique et d'Acoustique, 4 Impasse Nikola Tesla, CS 40006, 13453 Marseille Cedex 13, France.

Email: cocou@lma.cnrs-mrs.fr
} 
the adhesion, described by the intensity of adhesion introduced in [18, 19], were investigated in [31, 12, see also [34 and references therein.

Dynamic frictional contact problems with normal compliance laws have been studied in [24, 20, 4] and (non)local friction laws were considered in [21, 22, 16, 6, 13, for viscoelastic bodies. Dynamic frictionless problems with adhesion have been studied in [5, 23, 36] and dynamic viscoelastic problems coupling unilateral contact, recoverable adhesion and nonlocal friction have been analyzed in [14, 7].

Using the hemivariational inequalities theory, several nonsmooth quasistatic and dynamic contact problems were investigated, see [26, 27, 28, 29] and references therein. An elastic contact problem with relaxed unilateral conditions and pointwise Coulomb friction in the static case was studied in [30] and the extension to an elastic quasistatic contact problem was investigated in [10]. The corresponding viscoelastic dynamic case was analyzed in [7, 8, 9, 11] for different contact conditions.

Based on a new mixed variational formulation, given as a five-field evolution implicit equation coupled with a differential inclusion that describes the evolution of the intensity of adhesion, this work generalizes and extends the results presented in [7, 8, 9].

The results presented in this paper extend some of those proved in [11] in the more general case of rate-depending contact interactions, but with a limited adhesion (there is no intensity of adhesion), a linear viscosity operator and under stronger regularity assumptions.

The approach described in this paper enables to consider more general constitutive laws, as, for example, the ones characterizing some elastoviscoplastic materials investigated in [15].

The paper is organized as follows. In Section 2 the classical formulation of the dynamic contact problem is presented. In Section 3 two mixed variational formulations and some auxiliary results are given. Section 4 is devoted to the existence of a strong variational solution which is proved for an equivalent fixed point problem for a multi-valued function.

\section{Classical formulation}

We consider two viscoelastic bodies that occupy the reference domains $\Omega^{\alpha}$ of the three-dimensional Euclidean point space $\mathcal{E}$ with Lipschitz boundaries denoted by $\Gamma^{\alpha}=\partial \Omega^{\alpha}, \alpha=1,2$. Let $\Gamma_{U}^{\alpha}, \Gamma_{F}^{\alpha}$ and $\Gamma_{C}^{\alpha}$ denote three open disjoint sufficiently smooth parts of $\Gamma^{\alpha}$ such that $\Gamma^{\alpha}=\bar{\Gamma}_{U}^{\alpha} \cup \bar{\Gamma}_{F}^{\alpha} \cup \bar{\Gamma}_{C}^{\alpha}$ and, to simplify the estimates, meas $\left(\Gamma_{U}^{\alpha}\right)>0, \alpha=1,2$.

We assume the small deformation hypothesis and we use Cartesian coor- 
dinates representations with the summation convention for $i, j, k, l=1,2,3$. To simplify the presentation of the functional framework, we shall continue to use the vector and tensor notations for some inclusions.

Let $\boldsymbol{y}^{\alpha}\left(\boldsymbol{x}^{\alpha}, t\right)$ denote the position at time $t \in[0, T]$, where $0<T<+\infty$, of the material point $\boldsymbol{x}^{\alpha}$ represented by the coordinates $x^{\alpha}=\left(x_{1}^{\alpha}, x_{2}^{\alpha}, x_{3}^{\alpha}\right)$ in the reference configuration $\Omega^{\alpha}$, and $\boldsymbol{u}^{\alpha}\left(\boldsymbol{x}^{\alpha}, t\right)=\boldsymbol{y}^{\alpha}\left(\boldsymbol{x}^{\alpha}, t\right)-\boldsymbol{x}^{\alpha}$ denote the displacement vector of $\boldsymbol{x}^{\alpha}$ at time $t$, with the components $u^{\alpha}=\left(u_{1}^{\alpha}, u_{2}^{\alpha}, u_{3}^{\alpha}\right)$.

Let $\boldsymbol{\varepsilon}^{\alpha}$, with the components $\varepsilon^{\alpha}=\left(\varepsilon_{i j}\left(u^{\alpha}\right)\right)$, and $\boldsymbol{\sigma}^{\alpha}$, with the components $\sigma^{\alpha}=\left(\sigma_{i j}^{\alpha}\right)$, be the infinitesimal strain tensor and the stress tensor, respectively, corresponding to $\Omega^{\alpha}, \alpha=1,2$.

Denote by $\left(\mathbb{S}, \cdot,\|\cdot\|_{\mathbb{S}}\right)$ the space of symmetric second-order tensors with its inner product and the associated norm.

Let $\mathbf{A}^{\alpha}: \Omega^{\alpha} \times \mathbb{S} \rightarrow \mathbb{S}$ be the linear elasticity tensor corresponding to $\Omega^{\alpha}$ and denote by $A^{\alpha}=\left(A_{i j k l}^{\alpha}\right)$ its components satisfying the following classical symmetry and ellipticity conditions: $A_{i j k l}^{\alpha}=A_{j i k l}^{\alpha}=A_{k l i j}^{\alpha} \in$ $L^{\infty}\left(\Omega^{\alpha}\right), \quad \forall i, j, k, l=1,2,3, \exists k_{1}^{\alpha}>0$ such that $A_{i j k l}^{\alpha} \tau_{i j} \tau_{k l} \geq k_{1}^{\alpha} \tau_{i j} \tau_{i j}$ $\forall \tau=\left(\tau_{i j}\right) \in \mathbb{R}^{9}$ verifying $\tau_{i j}=\tau_{j i} \forall i, j=1,2,3, \alpha=1,2$. Thus, for all $\boldsymbol{\tau} \in \mathbb{S}$ with components $\tau=\left(\tau_{i j}\right), \mathbf{A}^{\alpha}\left(\boldsymbol{x}^{\alpha}, \boldsymbol{\tau}\right)$ has the components $A^{\alpha}\left(x^{\alpha}\right)_{i j k l} \tau_{i j} \forall k, l=1,2,3, \alpha=1,2$, and $\mathbf{A}^{\alpha}$ satisfies the following conditions for $\alpha=1,2$ :

$$
\left(\mathbf{A}^{\alpha}\left(\boldsymbol{x}^{\alpha}, \boldsymbol{\tau}\right)\right) \cdot \boldsymbol{\tau} \geq k_{1}^{\alpha}\|\boldsymbol{\tau}\|_{\mathbb{S}}^{2} \quad \text { a.e. } \boldsymbol{x}^{\alpha} \in \Omega^{\alpha}, \forall \boldsymbol{\tau} \in \mathbb{S},
$$

$\exists k_{2}^{\alpha}>0$ such that

$$
\left\|\mathbf{A}^{\alpha}\left(\boldsymbol{x}^{\alpha}, \boldsymbol{\tau}\right)\right\|_{\mathbb{S}} \leq k_{2}^{\alpha}\|\boldsymbol{\tau}\|_{\mathbb{S}} \quad \text { a.e. } \boldsymbol{x}^{\alpha} \in \Omega^{\alpha}, \forall \boldsymbol{\tau} \in \mathbb{S} .
$$

Let $\mathbf{B}^{\alpha}: \Omega^{\alpha} \times \mathbb{S} \rightarrow \mathbb{S}$ denote the nonlinear viscosity tensor corresponding to $\Omega^{\alpha}$, satisfying the following conditions for $\alpha=1,2$ :

$$
\begin{aligned}
& \exists k_{3,4}^{\alpha}>0 \text { such that a.e. } \boldsymbol{x}^{\alpha} \in \Omega^{\alpha}, \forall \boldsymbol{\tau}_{1,2} \in \mathbb{S}, \\
& \left(\mathbf{B}^{\alpha}\left(\boldsymbol{x}^{\alpha}, \boldsymbol{\tau}_{1}\right)-\mathbf{B}^{\alpha}\left(\boldsymbol{x}^{\alpha}, \boldsymbol{\tau}_{2}\right)\right) \cdot\left(\boldsymbol{\tau}_{1}-\boldsymbol{\tau}_{2}\right) \geq k_{3}^{\alpha}\left\|\boldsymbol{\tau}_{1}-\boldsymbol{\tau}_{2}\right\|_{\mathbb{S}}^{2}, \\
& \left\|\mathbf{B}^{\alpha}\left(\boldsymbol{x}^{\alpha}, \boldsymbol{\tau}_{1}\right)-\mathbf{B}^{\alpha}\left(\boldsymbol{x}^{\alpha}, \boldsymbol{\tau}_{2}\right)\right\|_{\mathbb{S}} \leq k_{4}^{\alpha}\left\|\boldsymbol{\tau}_{1}-\boldsymbol{\tau}_{2}\right\|_{\mathbb{S}}, \\
& \mathbf{B}^{\alpha}(\cdot, \mathbf{0})=\mathbf{0}, \\
& \forall \boldsymbol{\tau} \in \mathbb{S}, \mathbf{B}^{\alpha}(\cdot, \boldsymbol{\tau}) \text { is measurable on } \Omega^{\alpha} .
\end{aligned}
$$

Assume that the displacements $\boldsymbol{u}^{\alpha}=\mathbf{0}$ on $\Gamma_{U}^{\alpha} \times(0, T), \alpha=1,2$, and that the densities of both bodies are equal to 1 . Let $\boldsymbol{f}_{1}=\left(\boldsymbol{f}_{1}^{1}, \boldsymbol{f}_{1}^{2}\right), \boldsymbol{f}_{2}=$ $\left(\boldsymbol{f}_{2}^{1}, \boldsymbol{f}_{2}^{2}\right)$ be the given densities of body forces in $\Omega^{1} \cup \Omega^{2}$ and of tractions on $\Gamma_{F}^{1} \cup \Gamma_{F}^{2}$, respectively. Let $\boldsymbol{u}_{0}=\left(\boldsymbol{u}_{0}^{1}, \boldsymbol{u}_{0}^{2}\right), \boldsymbol{u}_{1}=\left(\boldsymbol{u}_{1}^{1}, \boldsymbol{u}_{1}^{2}\right)$ denote the initial displacements and velocities of the bodies, respectively. 
Suppose that the solids can be in contact between the potential contact surfaces $\Gamma_{C}^{1}$ and $\Gamma_{C}^{2}$ which are parametrized by two $C^{1}$ functions, $\varphi^{1}, \varphi^{2}$, defined on an open and bounded subset $\Xi$ of $\mathbb{R}^{2}$, such that $\varphi^{1}(\xi)-\varphi^{2}(\xi) \geq$ $0 \forall \xi \in \Xi$ and each $\Gamma_{C}^{\alpha}$ is the graph of $\varphi^{\alpha}$ on $\Xi$ that is $\Gamma_{C}^{\alpha}=\left\{\left(\xi, \varphi^{\alpha}(\xi)\right) \in\right.$ $\left.\mathbb{R}^{3} ; \xi \in \Xi\right\}, \alpha=1,2$, see, e.g., 3]. Define an initial normalized gap between the two contact surfaces by

$$
g_{0}(\xi)=\frac{\varphi^{1}(\xi)-\varphi^{2}(\xi)}{\sqrt{1+\left|\nabla \varphi^{1}(\xi)\right|^{2}}} \forall \xi \in \Xi
$$

Let $\boldsymbol{n}^{\alpha}$ denote the unit outward normal vector to $\Gamma^{\alpha}, \alpha=1,2$.

We introduce the following notations for the normal and tangential components of a displacement field $\boldsymbol{v}^{\alpha}$, of the relative displacement corresponding to $\boldsymbol{v}:=\left(\boldsymbol{v}^{1}, \boldsymbol{v}^{2}\right)$ and of the stress vector $\boldsymbol{\sigma}^{\alpha} \boldsymbol{n}^{\alpha}$ on $\Gamma_{C}^{\alpha}, \alpha=1,2$, respectively:

$$
\begin{aligned}
& \boldsymbol{v}^{\alpha}(\xi, t):=\boldsymbol{v}^{\alpha}\left(\xi, \varphi^{\alpha}(\xi), t\right), v_{N}^{\alpha}(\xi, t):=\boldsymbol{v}^{\alpha}(\xi, t) \cdot \boldsymbol{n}^{\alpha}(\xi), \\
& v_{N}(\xi, t):=v_{N}^{1}(\xi, t)+v_{N}^{2}(\xi, t), \\
& {\left[v_{N}\right](\xi, t):=v_{N}(\xi, t)-g_{0}(\xi),} \\
& \boldsymbol{v}_{T}^{\alpha}(\xi, t):=\boldsymbol{v}^{\alpha}(\xi, t)-v_{N}^{\alpha}(\xi, t) \boldsymbol{n}^{\alpha}(\xi), \\
& \boldsymbol{v}_{T}(\xi, t):=\boldsymbol{v}_{T}^{1}(\xi, t)-\boldsymbol{v}_{T}^{2}(\xi, t), \\
& \sigma_{N}^{\alpha}(\xi, t):=\left(\boldsymbol{\sigma}^{\alpha}(\xi, t) \boldsymbol{n}^{\alpha}(\xi)\right) \cdot \boldsymbol{n}^{\alpha}(\xi), \\
& \boldsymbol{\sigma}_{T}^{\alpha}(\xi, t)=\boldsymbol{\sigma}^{\alpha}(\xi, t) \boldsymbol{n}^{\alpha}(\xi)-\sigma_{N}^{\alpha}(\xi, t) \boldsymbol{n}^{\alpha}(\xi),
\end{aligned}
$$

for all $\xi \in \Xi$ and for all $t \in[0, T]$, where we denoted the inner product of two vectors by ". ".

In $\Xi$, we consider an internal state variable $\beta$ (see [18, 19]) that represents the intensity of adhesion: $\beta=1$ means that the adhesion is total, $\beta=0$ means that there is no adhesion and $0<\beta<1$ is the case of partial adhesion. We assume that the evolution of $\beta$ is governed, for all $t \in(0, T)$, by the inclusion $\dot{\beta} \in \psi\left(\left[u_{N}\right], \beta\right)$ in $\Xi$, where $\psi$ is a given constitutive set-valued mapping. Denote by $\beta_{0}$ the initial intensity of adhesion.

Let $\underline{\kappa}, \bar{\kappa}: \mathbb{R}^{2} \rightarrow \mathbb{R}$ be two mappings with $\underline{\kappa}$ lower semicontinuous and $\bar{\kappa}$ upper semicontinuous, satisfying the following conditions for all $s \in \mathbb{R}^{2}$ :

$$
\begin{aligned}
& \underline{\kappa}(s) \leq \bar{\kappa}(s) \text { and } 0 \notin(\underline{\kappa}(s), \bar{\kappa}(s)), \\
& \exists r_{0} \geq 0 \text { such that } \max (|\underline{\kappa}(s)|,|\bar{\kappa}(s)|) \leq r_{0},
\end{aligned}
$$

Let $\mu: \Xi \times \mathbb{R}^{3} \rightarrow \mathbb{R}_{+}$be the sliding velocity dependent coefficient of friction and assume that $\mu$ is a bounded function such that for a.e. $\xi \in \Xi$ $\mu(\xi, \cdot)$ is Lipschitz continuous with the Lipschitz constant independent of $\xi$, 
and for every $\boldsymbol{v} \in \mathbb{R}^{3} \mu(\cdot, \boldsymbol{v})$ is measurable. Define a truncation operator $\vartheta=\vartheta_{l_{0}}$ by $\vartheta: \mathbb{R} \rightarrow \mathbb{R}, \vartheta(s)=-l_{0}$ if $s \leq-l_{0}, \vartheta(s)=s$ if $|s|<l_{0}$ and $\vartheta(s)=l_{0}$ if $s \geq l_{0}$, where $l_{0}>0$ is a given characteristic length, see, e.g., [31, 36].

We choose the following state variables: the infinitesimal strain tensor $\left(\varepsilon^{1}, \boldsymbol{\varepsilon}^{2}\right)=\left(\boldsymbol{\varepsilon}\left(\boldsymbol{u}^{1}\right), \boldsymbol{\varepsilon}\left(\boldsymbol{u}^{2}\right)\right)$ in $\Omega^{1} \cup \Omega^{2}$, the normal relative displacement $\left[u_{N}\right]=$ $u_{N}^{1}+u_{N}^{2}-g_{0}$, the tangential relative displacement $\left[\boldsymbol{u}_{T}\right]=\boldsymbol{u}_{T}^{1}-\boldsymbol{u}_{T}^{2}$, and the intensity of adhesion $\beta$ in $\Xi$.

Consider the following dynamic viscoelastic contact problem coupling adhesion and Coulomb friction.

Problem $\boldsymbol{P}_{\boldsymbol{c}}$ : Find $\boldsymbol{u}=\left(\boldsymbol{u}^{1}, \boldsymbol{u}^{2}\right)$ and $\beta$ such that $\boldsymbol{u}(0)=\boldsymbol{u}_{0}, \dot{\boldsymbol{u}}(0)=\boldsymbol{u}_{1}$, $\beta(0)=\beta_{0}$ in $\Xi$ and, for all $t \in(0, T)$,

$$
\begin{aligned}
& \ddot{\boldsymbol{u}}^{\alpha}-\operatorname{div} \boldsymbol{\sigma}^{\alpha}\left(\boldsymbol{u}^{\alpha}, \dot{\boldsymbol{u}}^{\alpha}\right)=\boldsymbol{f}_{1}^{\alpha} \text { in } \Omega^{\alpha}, \\
& \boldsymbol{\sigma}^{\alpha}\left(\boldsymbol{u}^{\alpha}, \dot{\boldsymbol{u}}^{\alpha}\right)=\mathbf{A}^{\alpha} \boldsymbol{\varepsilon}\left(\boldsymbol{u}^{\alpha}\right)+\mathbf{B}^{\alpha} \boldsymbol{\varepsilon}\left(\dot{\boldsymbol{u}}^{\alpha}\right) \text { in } \Omega^{\alpha}, \\
& \boldsymbol{u}^{\alpha}=\mathbf{0} \text { on } \Gamma_{U}^{\alpha}, \boldsymbol{\sigma}^{\alpha} \boldsymbol{n}^{\alpha}=\boldsymbol{f}_{2}^{\alpha} \text { on } \Gamma_{F}^{\alpha}, \alpha=1,2, \\
& \boldsymbol{\sigma}^{1} \boldsymbol{n}^{1}+\boldsymbol{\sigma}^{2} \boldsymbol{n}^{2}=\mathbf{0} \text { in } \Xi, \\
& \underline{\kappa}\left(\left[u_{N}\right], \beta\right) \leq \sigma_{N} \leq \bar{\kappa}\left(\left[u_{N}\right], \beta\right) \text { in } \Xi, \\
& \left|\boldsymbol{\sigma}_{T}\right| \leq \mu\left(\dot{\boldsymbol{u}}_{T}\right)\left|\sigma_{N}\right| \text { in } \Xi \text { and } \\
& \quad \dot{\boldsymbol{u}}_{T} \neq \mathbf{0} \Rightarrow \boldsymbol{\sigma}_{T}=-\mu\left(\dot{\boldsymbol{u}}_{T}\right)\left|\sigma_{N}\right| \frac{\dot{\boldsymbol{u}}_{T}}{\left|\dot{\boldsymbol{u}}_{T}\right|}, \\
& \beta \in[0,1] \text { and } \dot{\beta} \in \psi\left(\vartheta\left(\left[u_{N}\right]\right), \beta\right) \text { in } \Xi,
\end{aligned}
$$

where, for all $\left(\boldsymbol{x}^{\alpha}, t\right) \in \Omega^{\alpha} \times(0, T)$,

$$
\begin{aligned}
& \mathbf{A}^{\alpha} \boldsymbol{\varepsilon}\left(\boldsymbol{u}^{\alpha}\right)\left(\boldsymbol{x}^{\alpha}, t\right)=\mathbf{A}^{\alpha}\left(\boldsymbol{x}^{\alpha}, \boldsymbol{\varepsilon}\left(\boldsymbol{u}^{\alpha}\left(\boldsymbol{x}^{\alpha}, t\right)\right)\right), \\
& \mathbf{B}^{\alpha} \boldsymbol{\varepsilon}\left(\dot{\boldsymbol{u}}^{\alpha}\right)\left(\boldsymbol{x}^{\alpha}, t\right)=\mathbf{B}^{\alpha}\left(\boldsymbol{x}^{\alpha}, \boldsymbol{\varepsilon}\left(\dot{\boldsymbol{u}}^{\alpha}\left(\boldsymbol{x}^{\alpha}, t\right)\right)\right), \\
& \boldsymbol{\sigma}^{\alpha}=\boldsymbol{\sigma}^{\alpha}\left(\boldsymbol{u}^{\alpha}, \dot{\boldsymbol{u}}^{\alpha}\right), \alpha=1,2, \sigma_{N}:=\sigma_{N}^{1}, \boldsymbol{\sigma}_{T}:=\boldsymbol{\sigma}_{T}^{1} .
\end{aligned}
$$

The nonlinear constitutive law represents a generalization of the classical Kelvin-Voigt law.

Different choices for $\underline{\kappa}, \bar{\kappa}$ and $\psi$ give various contact and friction conditions, including irreversible or recoverable (healing) adhesion, see [31, 12, 14, 9, 7].

We also remark that in this model friction and adhesion are strongly coupled, as sliding friction can occur even under tensile loads [25], but the case of the solely compressive loads can be easily considered. 


\section{Mixed variational formulations and approxi- mation results}

We adopt the following notations:

$$
\begin{aligned}
& \boldsymbol{H}^{s}\left(\Omega^{\alpha}\right):=H^{s}\left(\Omega^{\alpha} ; \mathbb{R}^{3}\right), \alpha=1,2, \\
& \boldsymbol{H}^{s}:=\boldsymbol{H}^{s}\left(\Omega^{1}\right) \times \boldsymbol{H}^{s}\left(\Omega^{2}\right) \forall s \in \mathbb{R}, \\
& \boldsymbol{V}^{\alpha}=\left\{\boldsymbol{v}^{\alpha} \in \boldsymbol{H}^{1}\left(\Omega^{\alpha}\right) ; \boldsymbol{v}^{\alpha}=\mathbf{0} \text { a.e. on } \Gamma_{U}^{\alpha}\right\}, \alpha=1,2, \\
& \boldsymbol{V}:=\boldsymbol{V}^{1} \times \boldsymbol{V}^{2}, \boldsymbol{H}:=\boldsymbol{H}^{0}=L^{2}\left(\Omega^{1} ; \mathbb{R}^{3}\right) \times L^{2}\left(\Omega^{2} ; \mathbb{R}^{3}\right) .
\end{aligned}
$$

$(\boldsymbol{H},||$.$) and (\boldsymbol{V},\|\|$.$) are Hilbert spaces with the associated inner products$ denoted by $(.,$.$) and by \langle.,$.$\rangle , respectively, \boldsymbol{V} \subset \boldsymbol{H} \subset \boldsymbol{V}^{\prime}$ with the inclusion mapping of $\boldsymbol{V}$ into $\boldsymbol{H}$ continuous and densely defined, where $\left(\boldsymbol{V}^{\prime},\|\cdot\|_{\boldsymbol{V}^{\prime}}\right)$ is the dual of $\boldsymbol{V}$ and $\boldsymbol{H}$ is identified with its own dual. Let $\langle., .\rangle_{\boldsymbol{V}^{\prime}, \boldsymbol{V}}$ denote the duality pairing between $\boldsymbol{V}^{\prime}$ and $\boldsymbol{V}$.

Define $\Xi_{T}=\Xi \times(0, T)$, the closed convex cones $L_{+}^{2}(\Xi), L_{+}^{2}\left(\Xi_{T}\right)$ and the closed convex set $L_{[0,1]}^{2}(\Xi)$ as follows:

$$
\begin{aligned}
& L_{+}^{2}(\Xi):=\left\{\delta \in L^{2}(\Xi) ; \delta \geq 0 \text { a.e. in } \Xi\right\}, \\
& L_{+}^{2}\left(\Xi_{T}\right):=\left\{\eta \in L^{2}\left(\Xi_{T}\right) ; \eta \geq 0 \text { a.e. in } \Xi_{T}\right\}, \\
& L_{[0,1]}^{2}(\Xi):=\left\{\delta \in L^{2}(\Xi) ; \delta \in[0,1] \text { a.e. in } \Xi\right\} .
\end{aligned}
$$

Let $\mathcal{A}: \boldsymbol{V} \rightarrow \boldsymbol{V}^{\prime}, \mathcal{B}: \boldsymbol{V} \rightarrow \boldsymbol{V}^{\prime}$ be two operators defined by

$$
\begin{aligned}
& \langle\mathcal{A} \boldsymbol{v}, \boldsymbol{w}\rangle_{\boldsymbol{V}^{\prime}, \boldsymbol{V}}=\sum_{\alpha=1,2} \int_{\Omega^{\alpha}}\left(\mathrm{A}^{\alpha} \boldsymbol{\varepsilon}\left(\boldsymbol{v}^{\alpha}\right)\right) \cdot \boldsymbol{\varepsilon}\left(\boldsymbol{w}^{\alpha}\right) d x, \\
& \langle\boldsymbol{B} \boldsymbol{v}, \boldsymbol{w}\rangle_{\boldsymbol{V}^{\prime}, \boldsymbol{V}}=\sum_{\alpha=1,2} \int_{\Omega^{\alpha}}\left(\mathbf{B}^{\alpha} \boldsymbol{\varepsilon}\left(\boldsymbol{v}^{\alpha}\right)\right) \cdot \boldsymbol{\varepsilon}\left(\boldsymbol{w}^{\alpha}\right) d x \\
& \forall \boldsymbol{v}=\left(\boldsymbol{v}^{1}, \boldsymbol{v}^{2}\right), \boldsymbol{w}=\left(\boldsymbol{w}^{1}, \boldsymbol{w}^{2}\right) \in \boldsymbol{V} .
\end{aligned}
$$

Under the above assumptions on $\mathbf{A}^{\alpha}, \mathbf{B}^{\alpha}, \alpha=1,2$, it follows that there exist $M_{\mathcal{A}}, M_{\mathcal{B}}$, such that, for all $\boldsymbol{v}, \boldsymbol{w} \in \boldsymbol{V}$,

$$
\|\mathcal{A} \boldsymbol{v}\|_{\boldsymbol{V}^{\prime}} \leq M_{\mathcal{A}}\|\boldsymbol{v}\|_{\boldsymbol{V}},\|\mathcal{B} \boldsymbol{v}-\mathcal{B} \boldsymbol{w}\|_{\boldsymbol{V}^{\prime}} \leq M_{\mathcal{B}}\|\boldsymbol{v}-\boldsymbol{w}\|_{\boldsymbol{V}}
$$

As meas $\left(\Gamma_{U}^{\alpha}\right)>0$, by using Korn's inequality it also follows that there exist $m_{\mathcal{A}}, m_{\mathcal{B}}>0$ such that, for all $\boldsymbol{v}, \boldsymbol{w} \in \boldsymbol{V}$,

$$
\begin{aligned}
& \langle\mathcal{A} \boldsymbol{v}, \boldsymbol{v}\rangle_{\boldsymbol{V}^{\prime}, \boldsymbol{V}} \geq m_{\mathcal{A}}\|\boldsymbol{v}\|_{\boldsymbol{V}}^{2} \\
& \langle\mathcal{B} \boldsymbol{v}-\mathcal{B} \boldsymbol{w}, \boldsymbol{v}-\boldsymbol{w}\rangle_{\boldsymbol{V}^{\prime}, \boldsymbol{V}} \geq m_{\mathcal{B}}\|\boldsymbol{v}-\boldsymbol{w}\|_{\boldsymbol{V}}^{2} .
\end{aligned}
$$


Assume $\boldsymbol{u}_{0} \in \boldsymbol{V}, \boldsymbol{u}_{1} \in \boldsymbol{H}, g_{0} \in L_{+}^{2}(\Xi), \boldsymbol{f}_{1}^{\alpha} \in L^{2}\left(0, T ; L^{2}\left(\Omega^{\alpha} ; \mathbb{R}^{3}\right)\right), \boldsymbol{f}_{2}^{\alpha} \in$ $L^{2}\left(0, T ; L^{2}\left(\Gamma_{F}^{\alpha} ; \mathbb{R}^{3}\right)\right), \alpha=1,2$, and define the mapping $\boldsymbol{f} \in L^{2}\left(0, T ; \boldsymbol{V}^{\prime}\right)$ by

$$
\begin{gathered}
\langle\boldsymbol{f}, \boldsymbol{v}\rangle_{\boldsymbol{V}^{\prime}, \boldsymbol{V}}=\sum_{\alpha=1,2} \int_{\Omega^{\alpha}} \boldsymbol{f}_{1}^{\alpha} \cdot \boldsymbol{v}^{\alpha} d x+\sum_{\alpha=1,2} \int_{\Gamma_{F}^{\alpha}} \boldsymbol{f}_{2}^{\alpha} \cdot \boldsymbol{v}^{\alpha} d s \\
\forall \boldsymbol{v}=\left(\boldsymbol{v}^{1}, \boldsymbol{v}^{2}\right) \in \boldsymbol{V}, \text { a.e. } t \in[0, T] .
\end{gathered}
$$

Assume also the following initial conditions: $\beta_{0} \in L_{[0,1]}^{2}(\Xi),\left[u_{0 N}\right] \leq 0$, and $\bar{\kappa}\left(\left[u_{0 N}\right], \beta_{0}\right)=0$ a.e. in $\Xi$.

For every $\zeta=\left(\zeta_{1}, \zeta_{2}\right) \in L^{2}\left(0, T ;\left(L^{2}(\Xi)\right)^{2}\right)=\left(L^{2}\left(\Xi_{T}\right)\right)^{2}$, define the following nonempty, closed, and convex sets:

$$
\begin{gathered}
\Lambda^{0}\left(\zeta_{1}, \zeta_{2}\right)=\left\{\eta \in L^{2}\left(\Xi_{T}\right) ; \underline{\kappa} \circ\left(\zeta_{1}, \zeta_{2}\right) \leq \eta\right. \\
\left.\leq \bar{\kappa} \circ\left(\zeta_{1}, \zeta_{2}\right) \text { a.e. in } \Xi_{T}\right\}, \\
\Lambda_{+}^{0}\left(\zeta_{1}, \zeta_{2}\right)=\left\{\eta \in L_{+}^{2}\left(\Xi_{T}\right) ; \underline{\kappa}_{+} \circ\left(\zeta_{1}, \zeta_{2}\right) \leq \eta\right. \\
\left.\leq \bar{\kappa}_{+} \circ\left(\zeta_{1}, \zeta_{2}\right) \text { a.e. in } \Xi_{T}\right\}, \\
\Lambda_{-}^{0}\left(\zeta_{1}, \zeta_{2}\right)=\left\{\eta \in L_{+}^{2}\left(\Xi_{T}\right) ; \bar{\kappa}_{-} \circ\left(\zeta_{1}, \zeta_{2}\right) \leq \eta\right. \\
\left.\leq \underline{\kappa}_{-} \circ\left(\zeta_{1}, \zeta_{2}\right) \text { a.e. in } \Xi_{T}\right\},
\end{gathered}
$$

where, for each $r \in \mathbb{R}, r_{+}:=\max (0, r)$ and $r_{-}:=\max (0,-r)$ denote the positive and negative parts, respectively. Also, for every $\boldsymbol{w} \in W^{1,2}(0, T ; \boldsymbol{V})$, $v \in L^{2}\left(\Xi_{T}\right)$, define the following nonempty and closed sets:

$$
\begin{aligned}
& \boldsymbol{\Lambda}^{1}(\boldsymbol{w}, v)=\left\{(\eta, \boldsymbol{\varsigma}) \in L^{2}\left(\Xi_{T}\right) \times\left(L^{2}\left(\Xi_{T}\right)\right)^{3} ;\right. \\
& \quad \eta \in \Lambda^{0}\left(\left[w_{N}\right], v\right),|\boldsymbol{\varsigma}| \leq \mu\left(\dot{\boldsymbol{w}}_{T}\right)|\eta|, \\
& \left.\quad \boldsymbol{\varsigma} \cdot \dot{\boldsymbol{w}}_{T}+\mu\left(\dot{\boldsymbol{w}}_{T}\right)|\eta|\left|\dot{\boldsymbol{w}}_{T}\right|=0 \text { a.e. in } \Xi_{T}\right\}, \\
& \boldsymbol{\Lambda}^{2}(\boldsymbol{w}, v)=\left\{(\eta, \boldsymbol{\varsigma}) \in L^{2}\left(\Xi_{T}\right) \times\left(L^{2}\left(\Xi_{T}\right)\right)^{3} ;\right. \\
& \quad \eta_{+} \in \Lambda_{+}^{0}\left(\left[w_{N}\right], v\right), \eta_{-} \in \Lambda_{-}^{0}\left(\left[w_{N}\right], v\right), \\
& \quad|\boldsymbol{\varsigma}| \leq \mu\left(\dot{\boldsymbol{w}}_{T}\right)\left(\eta_{+}+\eta_{-}\right), \\
& \left.\quad \boldsymbol{\varsigma} \cdot \dot{\boldsymbol{w}}_{T}+\mu\left(\dot{\boldsymbol{w}}_{T}\right)\left(\eta_{+}+\eta_{-}\right)\left|\dot{\boldsymbol{w}}_{T}\right|=0 \text { a.e. in } \Xi_{T}\right\}, \\
& \Lambda^{3}(\boldsymbol{w}, v)=\left\{\left(\eta_{1}, \eta_{2}, \boldsymbol{\varsigma}\right) \in\left(L^{2}\left(\Xi_{T}\right)\right)^{5} ; \eta_{1} \in \Lambda_{+}^{0}\left(\left[w_{N}\right], v\right),\right. \\
& \quad \eta_{2} \in \Lambda_{-}^{0}\left(\left[w_{N}\right], v\right),|\boldsymbol{\varsigma}| \leq \mu\left(\dot{\boldsymbol{w}}_{T}\right)\left(\eta_{1}+\eta_{2}\right), \\
& \left.\quad \boldsymbol{\varsigma} \cdot \dot{\boldsymbol{w}}_{T}+\mu\left(\dot{\boldsymbol{w}}_{T}\right)\left(\eta_{1}+\eta_{2}\right)\left|\dot{\boldsymbol{w}}_{T}\right|=0 \text { a.e. in } \Xi_{T}\right\} .
\end{aligned}
$$

The relations meas $(\Xi)<\infty$ and $(2.2)$ imply that for all $\zeta=\left(\zeta_{1}, \zeta_{2}\right) \in L^{2}\left(\Xi_{T}\right)$ the sets $\Lambda^{0}(\zeta), \Lambda_{+}^{0}(\zeta)$ and $\Lambda_{-}^{0}(\zeta)$ are bounded in norm in $L^{2}\left(0, T ; L^{2}(\Xi)\right)=L^{2}\left(\Xi_{T}\right)$ 
by $R_{0}=r_{0}(\operatorname{meas}(\Xi))^{1 / 2} T$ and are bounded in norm in $L^{\infty}\left(0, T ; L^{\infty}(\Xi)\right)$ by $r_{0}$.

As the coefficient of friction $\mu$ is a bounded function, it follows that for all $\boldsymbol{w} \in W^{1,2}(0, T ; \boldsymbol{V}), v \in L^{2}\left(\Xi_{T}\right)$ the sets $\boldsymbol{\Lambda}^{1}(\boldsymbol{w}, v), \boldsymbol{\Lambda}^{2}(\boldsymbol{w}, v)$, and $\boldsymbol{\Lambda}^{3}(\boldsymbol{w}, v)$ are bounded in norm. Thus, there exists $R_{1}>0$ such that $\boldsymbol{\Lambda}^{3}(\boldsymbol{w}) \subset D_{0} \times D_{1}$ for all $\boldsymbol{w} \in W^{1,2}(0, T ; \boldsymbol{V})$, where $D_{0}=\left\{\left(\eta_{1}, \eta_{2}\right) \in\left(L^{2}\left(\Xi_{T}\right)\right)^{2} ;\left\|\eta_{1}\right\|_{L^{2}\left(\Xi_{T}\right)} \leq\right.$ $\left.R_{0},\left\|\eta_{2}\right\|_{L^{2}\left(\Xi_{T}\right)} \leq R_{0}\right\}$ and $D_{1}=\left\{\varsigma \in\left(L^{2}\left(\Xi_{T}\right)\right)^{3} ;\|\varsigma\|_{\left(L^{2}\left(\Xi_{T}\right)\right)^{3}} \leq R_{1}\right\}$.

A first variational formulation of the problem $P_{c}$ is the following.

Problem $\boldsymbol{P}_{\boldsymbol{v}}^{\mathbf{1}}:$ Find $\boldsymbol{u} \in C^{1}([0, T] ; \boldsymbol{H}) \cap W^{1,2}(0, T ; \boldsymbol{V}) \cap W^{2,2}\left(0, T ; \boldsymbol{V}^{\prime}\right)$, $\lambda \in L^{2}\left(\Xi_{T}\right), \gamma \in\left(L^{2}\left(\Xi_{T}\right)\right)^{3}, \beta \in W^{1, \infty}\left(0, T ; L^{\infty}(\Xi)\right)$, such that $(\lambda, \gamma) \in$ $\Lambda^{1}(\boldsymbol{u}, \beta), \beta(t) \in L_{[0,1]}^{2}(\Xi)$ for all $t \in(0, T), \boldsymbol{u}(0)=\boldsymbol{u}_{0}, \dot{\boldsymbol{u}}(0)=\boldsymbol{u}_{1}, \beta(0)=$ $\beta_{0}$, and for almost all $t \in(0, T)$

$$
\begin{aligned}
& \langle\ddot{\boldsymbol{u}}, \boldsymbol{v}\rangle_{\boldsymbol{V}^{\prime}, \boldsymbol{V}}+\langle\mathcal{A} \boldsymbol{u}, \boldsymbol{v}\rangle_{\boldsymbol{V}^{\prime}, \boldsymbol{V}}+\langle\mathcal{B} \dot{u}, \boldsymbol{v}\rangle_{\boldsymbol{V}^{\prime}, \boldsymbol{V}} \\
& -\left(\lambda, v_{N}\right)_{L^{2}(\Xi)}-\left(\boldsymbol{\gamma}, \boldsymbol{v}_{T}\right)_{\left(L^{2}(\Xi)\right)^{3}}=\langle\boldsymbol{f}, \boldsymbol{v}\rangle_{\boldsymbol{V}^{\prime}, \boldsymbol{V}} \forall \boldsymbol{v} \in \boldsymbol{V}, \\
& \dot{\beta} \in \psi\left(\vartheta\left(\left[u_{N}\right]\right), \beta\right) \text { a.e. in } \Xi_{T},
\end{aligned}
$$

where $(\cdot, \cdot)_{L^{2}(\Xi)}$ and $(\cdot, \cdot)_{\left(L^{2}(\Xi)\right)^{3}}$ denote the inner products of the corresponding spaces.

The formal equivalence between the variational problem $P_{v}^{1}$ and the classical problem $P_{c}$ can be proved as usual by Green's formula, where the Lagrange multipliers $\lambda, \boldsymbol{\gamma}$ satisfy the relations $\lambda=\sigma_{N}, \boldsymbol{\gamma}=\boldsymbol{\sigma}_{T}$.

The sets $\Lambda^{0}\left(\zeta_{1}, \zeta_{2}\right), \Lambda_{+}^{0}\left(\zeta_{1}, \zeta_{2}\right)$ and $\Lambda_{-}^{0}\left(\zeta_{1}, \zeta_{2}\right)$ have the following useful properties, see [9].

Lemma 3.1. Let $\left(\zeta_{1}, \zeta_{2}\right) \in\left(L^{2}(\Xi)\right)^{2}$ and $\left(\eta_{1}, \eta_{2}\right) \in \Lambda_{+}^{0}\left(\zeta_{1}, \zeta_{2}\right) \times \Lambda_{-}^{0}\left(\zeta_{1}, \zeta_{2}\right)$. Then $\eta_{1} \eta_{2}=0$ a.e. in $\Xi_{T}$ and there exists $\eta \in \Lambda^{0}\left(\zeta_{1}, \zeta_{2}\right)$ such that $\eta_{+}=\eta_{1}$, $\eta_{-}=\eta_{2}$ a.e. in $\Xi_{T}$.

Since $\lambda \in \Lambda^{0}\left(\left[u_{N}\right], \beta\right)$ if and only if $\left(\lambda_{+}, \lambda_{-}\right) \in \Lambda_{+}^{0}\left(\left[u_{N}\right], \beta\right) \times \Lambda_{-}^{0}\left(\left[u_{N}\right], \beta\right)$, the previous lemma enables to consider the following variational problem $P_{v}^{2}$, which has the same solutions $\boldsymbol{u}, \gamma, \beta$ as the problem $P_{v}^{1}$ and the solutions $\lambda_{1}, \lambda_{2}$ satisfy the relation $\lambda=\lambda_{1}-\lambda_{2}$, where $\lambda$ is a solution of $P_{v}^{1}$.

Problem $\boldsymbol{P}_{\boldsymbol{v}}^{\mathbf{2}}$ : Find $\boldsymbol{u} \in C^{1}([0, T] ; \boldsymbol{H}) \cap W^{1,2}(0, T ; \boldsymbol{V}) \cap W^{2,2}\left(0, T ; \boldsymbol{V}^{\prime}\right)$, $\left(\lambda_{1}, \lambda_{2}\right) \in\left(L^{2}\left(\Xi_{T}\right)\right)^{2}, \gamma \in\left(L^{2}\left(\Xi_{T}\right)\right)^{3}, \beta \in W^{1, \infty}\left(0, T ; L^{\infty}(\Xi)\right)$, such that $\left(\lambda_{1}, \lambda_{2}, \gamma\right) \in \Lambda^{3}(\boldsymbol{u}, \beta), \beta(t) \in L_{[0,1]}^{2}(\Xi)$ for all $t \in(0, T), \boldsymbol{u}(0)=\boldsymbol{u}_{0}$, 
$\dot{\boldsymbol{u}}(0)=\boldsymbol{u}_{1}, \beta(0)=\beta_{0}$, and for almost all $t \in(0, T)$

$$
\begin{aligned}
\langle\ddot{\boldsymbol{u}}, \boldsymbol{v}\rangle_{\boldsymbol{V}^{\prime}, \boldsymbol{V}}+\langle\mathcal{A} \boldsymbol{u}, \boldsymbol{v}\rangle_{\boldsymbol{V}^{\prime}, \boldsymbol{V}}+\langle\mathcal{B} \dot{\boldsymbol{u}}, \boldsymbol{v}\rangle_{\boldsymbol{V}^{\prime}, \boldsymbol{V}} \\
-\left(\lambda_{1}-\lambda_{2}, v_{N}\right)_{L^{2}(\Xi)}-\left(\boldsymbol{\gamma}, \boldsymbol{v}_{T}\right)_{\left(L^{2}(\Xi)\right)^{3}} \\
\quad=\langle\boldsymbol{f}, \boldsymbol{v}\rangle_{\boldsymbol{V}^{\prime}, \boldsymbol{V}} \quad \forall \boldsymbol{v} \in \boldsymbol{V}, \\
\dot{\beta} \in \psi\left(\vartheta\left(\left[u_{N}\right]\right), \beta\right) \quad \text { a.e. in } \Xi_{T},
\end{aligned}
$$

Assume that the set-valued mapping $\psi:\left(L^{2}\left(\Xi_{T}\right)\right)^{2} \rightarrow 2^{L^{2}\left(\Xi_{T}\right)}$ verifies the following properties: for each $\boldsymbol{u} \in W^{1,2}(0, T ; \boldsymbol{V})$ there exists a unique solution $\beta_{\boldsymbol{u}} \in W^{1, \infty}\left(0, T ; L^{\infty}(\Xi)\right)$ of the inclusion (3.6), such that $\beta(0)=\beta_{0}$, $\beta(t) \in L_{[0,1]}^{2}(\Xi)$ for all $t \in(0, T)$, and if $\beta_{\boldsymbol{u}_{1}}, \beta_{\boldsymbol{u}_{2}}$ are the solutions of (3.6) corresponding to $\boldsymbol{u}_{1}, \boldsymbol{u}_{2} \in W^{1,2}(0, T ; \boldsymbol{V})$, respectively, with the same initial condition $\beta_{0}$, then the following estimate holds for all $t \in[0, T]$ :

$$
\left\|\beta_{u_{1}}(t)-\beta_{u_{2}}(t)\right\|_{L^{2}(\Xi)}^{2} \leq C_{0} \int_{0}^{t}\left\|\boldsymbol{u}_{1}(s)-\boldsymbol{u}_{2}(s)\right\|^{2} d s
$$

where $C_{0}$ is a positive constant independent of $\boldsymbol{u}_{1}, \boldsymbol{u}_{2}, \beta_{\boldsymbol{u}_{1}}, \beta_{\boldsymbol{u}_{2}}$.

Several choices of $\psi$, as subdifferentials or single-valued mappings satisfying the previous properties, have been considered, see, e.g., [31, 34, 14, 7].

The existence of strong solutions to problem $P_{v}^{2}$ will be established by using some auxiliary results and an equivalent fixed point problem.

Similar arguments to those used to prove Theorem 5.2 in [36, now applied to the product space $\boldsymbol{V}=\boldsymbol{V}^{1} \times \boldsymbol{V}^{2}$ and to the simple case without adhesion, enable to obtain the following existence and uniqueness result for the strong solution, in the $\boldsymbol{V}^{\prime}$ sense, of the following intermediate problem.

Lemma 3.2. For each $\left(\eta_{1}, \eta_{2}\right) \in\left(L^{2}\left(\Xi_{T}\right)\right)^{2}, \boldsymbol{\varsigma} \in\left(L^{2}\left(\Xi_{T}\right)\right)^{3}$, there exists a unique function $\boldsymbol{u}=\boldsymbol{u}_{\left(\eta_{1}, \eta_{2}, \boldsymbol{\varsigma}\right)} \in C^{1}([0, T] ; \boldsymbol{H}) \cap W^{1,2}(0, T ; \boldsymbol{V}) \cap W^{2,2}\left(0, T ; \boldsymbol{V}^{\prime}\right)$, such that $\boldsymbol{u}(0)=\boldsymbol{u}_{0}, \dot{\boldsymbol{u}}(0)=\boldsymbol{u}_{1}$, and for almost all $t \in(0, T)$

$$
\begin{aligned}
& \langle\ddot{\boldsymbol{u}}, \boldsymbol{v}\rangle_{\boldsymbol{V}^{\prime}, \boldsymbol{V}}+\langle\mathcal{A} \boldsymbol{u}, \boldsymbol{v}\rangle_{\boldsymbol{V}^{\prime}, \boldsymbol{V}}+\langle\mathcal{B} \dot{\boldsymbol{u}}, \boldsymbol{v}\rangle_{\boldsymbol{V}^{\prime}, \boldsymbol{V}} \\
& -\left(\eta_{1}-\eta_{2}, v_{N}\right)_{L^{2}(\Xi)}-\left(\boldsymbol{\varsigma}, \boldsymbol{v}_{T}\right)_{\left(L^{2}(\Xi)\right)^{3}} \\
& =\langle\boldsymbol{f}, \boldsymbol{v}\rangle_{\boldsymbol{V}^{\prime}, \boldsymbol{V}} \quad \forall \boldsymbol{v} \in \boldsymbol{V}
\end{aligned}
$$

where $\ddot{\boldsymbol{u}}$ is the second order strong derivative of $\boldsymbol{u}$ considered in $\boldsymbol{V}^{\prime}$.

For the involved abstract mathematical result, see, e.g., Theorem 4.10 in [2]. 
Lemma 3.3. Let $\boldsymbol{u}_{\left(\eta_{1}, \eta_{2}, \varsigma_{1}\right)}, \boldsymbol{u}_{\left(\delta_{1}, \delta_{2}, \varsigma_{2}\right)}$ be the solutions of variational equation (3.8) corresponding to $\left(\eta_{1}, \eta_{2}\right),\left(\delta_{1}, \delta_{2}\right) \in\left(L^{2}\left(\Xi_{T}\right)\right)^{2}, \varsigma_{1}, \varsigma_{2} \in\left(L^{2}\left(\Xi_{T}\right)\right)^{3}$, respectively.

Then there exists a constant $C_{1}>0$, independent of $\left(\eta_{1}, \eta_{2}\right),\left(\delta_{1}, \delta_{2}\right)$, and $\varsigma_{1}, \varsigma_{2}$, such that for all $t \in[0, T]$

$$
\begin{aligned}
& \left|\dot{\boldsymbol{u}}_{\left(\eta_{1}, \eta_{2}, \boldsymbol{\varsigma}_{1}\right)}(t)-\dot{\boldsymbol{u}}_{\left(\delta_{1}, \delta_{2}, \boldsymbol{\varsigma}_{2}\right)}(t)\right|^{2}+\left\|\boldsymbol{u}_{\left(\eta_{1}, \eta_{2}, \boldsymbol{\varsigma}_{1}\right)}(t)-\boldsymbol{u}_{\left(\delta_{1}, \delta_{2}, \boldsymbol{\varsigma}_{2}\right)}(t)\right\|^{2} \\
& \quad+\int_{0}^{t}\left\|\dot{\boldsymbol{u}}_{\left(\eta_{1}, \eta_{2}, \boldsymbol{\varsigma}_{1}\right)}-\dot{\boldsymbol{u}}_{\left(\delta_{1}, \delta_{2}, \boldsymbol{\varsigma}_{2}\right)}\right\|^{2} d \tau \\
& \leq C_{1} \int_{0}^{t}\left\{\left(\eta_{1}-\eta_{2}-\delta_{1}+\delta_{2}, \dot{u}_{\left(\eta_{1}, \eta_{2}, \boldsymbol{\varsigma}_{1}\right) N}-\dot{u}_{\left(\delta_{1}, \delta_{2}, \boldsymbol{\varsigma}_{2}\right) N}\right)_{L^{2}(\Xi)}\right. \\
& \left.\quad+\left(\boldsymbol{\varsigma}_{1}-\boldsymbol{\varsigma}_{2}, \dot{\boldsymbol{u}}_{\left(\eta_{1}, \eta_{2}, \boldsymbol{\varsigma}_{1}\right) T}-\dot{\boldsymbol{u}}_{\left(\delta_{1}, \delta_{2}, \boldsymbol{\varsigma}_{2}\right) T}\right)_{\left(L^{2}(\Xi)\right)^{3}}\right\} d \tau
\end{aligned}
$$

Proof. Let $\left(\eta_{1}, \eta_{2}\right),\left(\delta_{1}, \delta_{2}\right) \in\left(L^{2}\left(\Xi_{T}\right)\right)^{2}$ and $\boldsymbol{\varsigma}_{1}, \boldsymbol{\varsigma}_{2} \in\left(L^{2}\left(\Xi_{T}\right)\right)^{3}$ with $\boldsymbol{u}_{1}:=$ $\boldsymbol{u}_{\left(\eta_{1}, \eta_{2}, \boldsymbol{\varsigma}_{1}\right)}, \boldsymbol{u}_{2}:=\boldsymbol{u}_{\left(\delta_{1}, \delta_{2}, \boldsymbol{\varsigma}_{2}\right)}$ the corresponding solutions of 3.8 which exist according to Lemma 3.2 . Taking in each equation $\boldsymbol{v}=\dot{\boldsymbol{u}}_{1}-\dot{\boldsymbol{u}}_{2}$, for a.e. $\tau \in(0, T)$ it follows that

$$
\begin{aligned}
\left\langle\ddot{\boldsymbol{u}}_{1}-\right. & \left.\ddot{\boldsymbol{u}}_{2}, \dot{\boldsymbol{u}}_{1}-\dot{\boldsymbol{u}}_{2}\right\rangle_{\boldsymbol{V}^{\prime}, \boldsymbol{V}}+\left\langle\mathcal{A} \boldsymbol{u}_{1}-\mathcal{A} \boldsymbol{u}_{2}, \dot{\boldsymbol{u}}_{1}-\dot{\boldsymbol{u}}_{2}\right\rangle_{\boldsymbol{V}^{\prime}, \boldsymbol{V}} \\
& +\left\langle\mathcal{B} \dot{\boldsymbol{u}}_{1}-\boldsymbol{B} \dot{\boldsymbol{u}}_{2}, \dot{\boldsymbol{u}}_{1}-\dot{\boldsymbol{u}}_{2}\right\rangle_{\boldsymbol{V}^{\prime}, \boldsymbol{V}} \\
& =\left(\eta_{1}-\eta_{2}-\delta_{1}+\delta_{2}, \dot{u}_{1 N}-\dot{u}_{2 N}\right)_{L^{2}(\Xi)} \\
& +\left(\boldsymbol{\varsigma}_{1}-\boldsymbol{\varsigma}_{2}, \dot{\boldsymbol{u}}_{1 T}-\dot{\boldsymbol{u}}_{2 T}\right)_{\left(L^{2}(\Xi)\right)^{3}} .
\end{aligned}
$$

Since the solutions $\boldsymbol{u}_{1}, \boldsymbol{u}_{2}$ belong to $\boldsymbol{u} \in C^{1}([0, T] ; \boldsymbol{H}) \cap W^{1,2}(0, T ; \boldsymbol{V}) \cap$ $W^{2,2}\left(0, T ; \boldsymbol{V}^{\prime}\right)$ and verify the same initial conditions, by integrating over $(0, t)$ it follows that for all $t \in[0, T]$

$$
\begin{aligned}
& \frac{1}{2}\left|\dot{\boldsymbol{u}}_{1}(t)-\dot{\boldsymbol{u}}_{2}(t)\right|^{2}+\frac{1}{2}\left\langle\mathcal{A}\left(\boldsymbol{u}_{1}-\boldsymbol{u}_{2}\right), \boldsymbol{u}_{1}-\boldsymbol{u}_{2}\right\rangle_{\boldsymbol{V}^{\prime}, \boldsymbol{V}} \\
& +\int_{0}^{t}\left\langle\boldsymbol{B} \dot{\boldsymbol{u}}_{1}-\boldsymbol{B} \dot{\boldsymbol{u}}_{2}, \dot{\boldsymbol{u}}_{1}-\dot{\boldsymbol{u}}_{2}\right\rangle_{\boldsymbol{V}^{\prime}, \boldsymbol{V}} d \tau \\
& =\int_{0}^{t}\left\{\left(\eta_{1}-\eta_{2}-\delta_{1}+\delta_{2}, \dot{u}_{1 N}-\dot{u}_{2 N}\right)_{L^{2}(\Xi)}\right\} d \tau \\
& +\int_{0}^{t}\left\{\left(\boldsymbol{\varsigma}_{1}-\boldsymbol{\varsigma}_{2}, \dot{\boldsymbol{u}}_{1 T}-\dot{\boldsymbol{u}}_{2 T}\right)_{\left(L^{2}(\Xi)\right)^{3}}\right\} d \tau .
\end{aligned}
$$

By $(3.2)$ and (3.3), the estimate $(3.9)$ follows. 
Lemma 3.4. Under the assumptions of Sections 2 and 3, for every $\left(\eta_{1}, \eta_{2}\right) \in$ $\left(L_{+}^{2}\left(\Xi_{T}\right)\right)^{2}$ and every $\varsigma \in\left(L^{2}\left(\Xi_{T}\right)\right)^{3}$, let $\left(\eta_{1}^{n}, \eta_{2}^{n}\right)_{n}$ be a sequence in $\left(L_{+}^{2}\left(\Xi_{T}\right)\right)^{2}$ and $\left(\varsigma^{n}\right)_{n}$ be a sequence in $\left(L^{2}\left(\Xi_{T}\right)\right)^{3}$ such that $\eta_{1}^{n} \rightarrow \eta_{1}, \eta_{2}^{n} \rightarrow \eta_{2}$ in $L^{2}\left(\Xi_{T}\right)$, and $\boldsymbol{\varsigma}^{n} \rightarrow \boldsymbol{\varsigma}$ in $\left(L^{2}\left(\Xi_{T}\right)\right)^{3}$. Let $\boldsymbol{u}_{\left(\eta_{1}^{n}, \eta_{2}^{n}, \boldsymbol{\varsigma}^{n}\right)}$ be the solution of (3.8) corresponding to $\left(\eta_{1}^{n}, \eta_{2}^{n}, \varsigma^{n}\right)$ according to Lemma 3.2. for every $n \in \mathbb{N}$. Then $\left(\boldsymbol{u}_{\left(\eta_{1}^{n}, \eta_{2}^{n}, \varsigma^{n}\right)}\right)_{n}$ is strongly convergent in $C^{1}([0, T] ; \boldsymbol{H}) \cap W^{1,2}(0, T ; \boldsymbol{V})$ to the solution $\boldsymbol{u}=\boldsymbol{u}_{\left(\eta_{1}, \eta_{2}, \boldsymbol{\varsigma}\right)}$ of (3.8) corresponding to $\left(\eta_{1}, \eta_{2}, \boldsymbol{\varsigma}\right)$ according to Lemma 3.2.

Proof. We adopt the following notations:

$$
\boldsymbol{u}_{n}:=\boldsymbol{u}_{\left(\eta_{1}^{n}, \eta_{2}^{n}, \varsigma^{n}\right)}, u_{n N}:=u_{\left(\eta_{1}^{n}, \eta_{2}^{n}, \varsigma^{n}\right) N}
$$

so that we have for almost all $t \in(0, T)$

$$
\begin{aligned}
& \left\langle\ddot{\boldsymbol{u}}_{n}, \boldsymbol{v}\right\rangle_{\boldsymbol{V}^{\prime}, \boldsymbol{V}}+\left\langle\mathcal{A} \boldsymbol{u}_{n}, \boldsymbol{v}\right\rangle_{\boldsymbol{V}^{\prime}, \boldsymbol{V}}+\left\langle\mathcal{B} \dot{\boldsymbol{u}}_{n}, \boldsymbol{v}\right\rangle_{\boldsymbol{V}^{\prime}, \boldsymbol{V}} \\
& -\left(\eta_{1}^{n}-\eta_{2}^{n}, v_{N}\right)_{L^{2}(\Xi)}-\left(\boldsymbol{\varsigma}^{n}, \boldsymbol{v}_{T}\right)_{\left(L^{2}(\Xi)\right)^{3}} \\
& \quad=\langle\boldsymbol{f}, \boldsymbol{v}\rangle_{\boldsymbol{V}^{\prime}, \boldsymbol{V}} \quad \forall \boldsymbol{v} \in \boldsymbol{V} .
\end{aligned}
$$

By Lemma 3.3, for all $n \in \mathbb{N}$ and $t \in(0, T]$ we have

$$
\begin{aligned}
& \frac{1}{2}\left|\dot{\boldsymbol{u}}_{n}(t)\right|^{2}+\frac{1}{2}\left\|\boldsymbol{u}_{n}(t)\right\|^{2}+\frac{1}{2} \int_{0}^{t}\left\|\dot{\boldsymbol{u}}_{n}\right\|^{2} d \tau \\
\leq & \left|\dot{\boldsymbol{u}}_{n}(t)-\dot{\boldsymbol{u}}(t)\right|^{2}+\left\|\boldsymbol{u}_{n}(t)-\boldsymbol{u}(t)\right\|^{2}+\int_{0}^{t}\left\|\dot{\boldsymbol{u}}_{n}-\dot{\boldsymbol{u}}\right\|^{2} d \tau \\
& +|\dot{\boldsymbol{u}}(t)|^{2}+\|\boldsymbol{u}(t)\|^{2}+\int_{0}^{t}\|\dot{\boldsymbol{u}}\|^{2} d \tau \\
\leq & C_{1} \int_{0}^{t}\left\{\left(\eta_{1}^{n}-\eta_{2}^{n}-\eta_{1}+\eta_{2}, \dot{u}_{n N}-\dot{u}_{N}\right)_{L^{2}(\Xi)}\right. \\
& \left.+\left(\boldsymbol{\varsigma}^{n}-\boldsymbol{\varsigma}, \dot{\boldsymbol{u}}_{n T}-\dot{\boldsymbol{u}}_{T}\right)_{\left(L^{2}(\Xi)\right)^{3}}\right\} d \tau+C_{2},
\end{aligned}
$$

where $C_{2}$ is a positive constant dependent only on $\boldsymbol{u}$.

Since the sequences $\left(\eta_{1}^{n}, \eta_{2}^{n}\right)_{n},\left(\varsigma^{n}\right)_{n}$ are bounded in $\left(L^{2}\left(\Xi_{T}\right)\right)^{2},\left(L^{2}\left(\Xi_{T}\right)\right)^{3}$, respectively, by Young's inequality it follows that there exists a positive constant $C_{3}$, depending only on $\boldsymbol{u}, C_{1}, C_{2}$, the bounds of $\left(\eta_{1}^{n}, \eta_{2}^{n}\right)_{n}$ and $\left(\boldsymbol{\varsigma}^{n}\right)_{n}$, such that the following estimates hold for all $n \in \mathbb{N}$ :

$$
\left|\dot{\boldsymbol{u}}_{n}(t)\right| \leq C_{3},\left\|\boldsymbol{u}_{n}(t)\right\| \leq C_{3} \quad \forall t \in[0, T],\left\|\dot{\boldsymbol{u}}_{n}\right\|_{L^{2}(0, T ; \boldsymbol{V})} \leq C_{3}
$$


For all $\boldsymbol{v} \in L^{2}(0, T ; \boldsymbol{V})$, by 3.10 we have

$$
\begin{aligned}
& \int_{0}^{T}\left\langle\ddot{\boldsymbol{u}}_{n}, \boldsymbol{v}\right\rangle_{\boldsymbol{V}^{\prime}, \boldsymbol{V}} d t+\int_{0}^{T}\left\langle\mathcal{A} \boldsymbol{u}_{n}, \boldsymbol{v}\right\rangle_{\boldsymbol{V}^{\prime}, \boldsymbol{V}} d t \\
& +\int_{0}^{T}\left\langle\boldsymbol{B} \dot{\boldsymbol{u}}_{n}, \boldsymbol{v}\right\rangle_{\boldsymbol{V}^{\prime}, \boldsymbol{V}} d t-\int_{0}^{T}\left(\eta_{1}^{n}-\eta_{2}^{n}, v_{N}\right)_{L^{2}(\Xi)} d t \\
& -\int_{0}^{T}\left(\boldsymbol{\varsigma}^{n}, \boldsymbol{v}_{T}\right)_{\left(L^{2}(\Xi)\right)^{3}} d t=\int_{0}^{T}\langle\boldsymbol{f}, \boldsymbol{v}\rangle_{\boldsymbol{V}^{\prime}, \boldsymbol{V}} d t
\end{aligned}
$$

This relation and the estimates (3.1), (3.11) imply that there exists a positive constant $C_{4}$, depending only on $C_{3}, M_{\mathcal{A}}$, and $M_{\mathcal{B}}$, such that

$$
\forall n \in \mathbb{N}, \quad\left\|\ddot{\boldsymbol{u}}_{n}\right\|_{L^{2}\left(0, T ; \boldsymbol{V}^{\prime}\right)} \leq C_{4} .
$$

From (3.11), (3.12), it follows that there exist a subsequence $\left(\boldsymbol{u}_{n_{k}}\right)_{k}$ and $\tilde{\boldsymbol{u}}$ such that

$$
\begin{aligned}
& \dot{\boldsymbol{u}}_{n_{k}} \rightarrow^{*} \dot{\tilde{\boldsymbol{u}}} \text { in } L^{\infty}(0, T ; \boldsymbol{H}), \quad \boldsymbol{u}_{n_{k}} \rightarrow^{*} \tilde{\boldsymbol{u}} \text { in } L^{\infty}(0, T ; \boldsymbol{V}), \\
& \dot{\boldsymbol{u}}_{n_{k}} \rightarrow \dot{\tilde{\boldsymbol{u}}} \text { in } L^{2}(0, T ; \boldsymbol{V}), \quad \ddot{\boldsymbol{u}}_{n_{k}} \rightarrow \ddot{\tilde{\boldsymbol{u}}} \text { in } L^{2}\left(0, T ; \boldsymbol{V}^{\prime}\right) .
\end{aligned}
$$

Since $\boldsymbol{V} \subset \boldsymbol{H}^{\iota} \subset \boldsymbol{H} \subset \boldsymbol{V}^{\prime}$ with compact embedding from $\boldsymbol{V}$ into $\boldsymbol{H}^{\iota}$, according to a classical compactness result, see, e.g., [35], it follows that

$$
\dot{\boldsymbol{u}}_{n_{k}} \rightarrow \dot{\tilde{\boldsymbol{u}}} \text { in } L^{2}\left(0, T ; \boldsymbol{H}^{\iota}\right),
$$

where $1>\iota>\frac{1}{2}$, so that, by the trace theorem,

$$
\dot{\boldsymbol{u}}_{n_{k}} \rightarrow \dot{\tilde{\boldsymbol{u}}} \text { in } L^{2}\left(0, T ;\left(L^{2}(\Xi)\right)^{3}\right)=\left(L^{2}\left(\Xi_{T}\right)\right)^{3} .
$$

By Lemma 3.3, for all $k \in \mathbb{N}$ and $t \in(0, T]$ we have

$$
\begin{aligned}
& \left|\dot{\boldsymbol{u}}_{n_{k}}(t)-\dot{\boldsymbol{u}}(t)\right|^{2}+\left\|\boldsymbol{u}_{n_{k}}(t)-\boldsymbol{u}(t)\right\|^{2}+\int_{0}^{t}\left\|\dot{\boldsymbol{u}}_{n_{k}}-\dot{\boldsymbol{u}}\right\|^{2} d \tau \\
& \leq C_{1} \int_{0}^{t}\left\{\left(\eta_{1}^{n_{k}}-\eta_{2}^{n_{k}}-\eta_{1}+\eta_{2}, \dot{u}_{n_{k} N}-\dot{u}_{N}\right)_{L^{2}(\Xi)}\right. \\
& \left.\quad+\left(\boldsymbol{\varsigma}^{n_{k}}-\boldsymbol{\varsigma}, \dot{\boldsymbol{u}}_{n_{k} T}-\dot{\boldsymbol{u}}_{T}\right)_{\left(L^{2}(\Xi)\right)^{3}}\right\} d \tau+C_{2},
\end{aligned}
$$

Using the weak convergence properties of $\left(\eta_{1}^{n}\right)_{n},\left(\eta_{2}^{n}\right)_{n},\left(\varsigma^{n}\right)_{n}$, and the strong convergence property (3.13), we can pass to limits in the right hand side of the previous estimates for $\left(\boldsymbol{u}_{n_{k}}\right)_{k}$ and so we obtain that $\boldsymbol{u}=\tilde{\boldsymbol{u}}$ and

$$
\boldsymbol{u}_{n_{k}} \rightarrow \boldsymbol{u} \text { in } C^{1}([0, T] ; \boldsymbol{H}) \cap W^{1,2}(0, T ; \boldsymbol{V}) .
$$


As for every subsequence of $\left(\boldsymbol{u}_{n}\right)_{n}$, by using the same arguments as above that enabled to obtain the relation (3.14), one can always find a (sub)subsequence converging to $\boldsymbol{u}$ in $C^{1}([0, T] ; \boldsymbol{H}) \cap W^{1,2}(0, T ; \boldsymbol{V})$, it follows that

$$
\boldsymbol{u}_{\left(\eta_{1}^{n}, \eta_{2}^{n}, \varsigma^{n}\right)} \rightarrow \boldsymbol{u}_{\left(\eta_{1}, \eta_{2}, \varsigma\right)} \text { in } C^{1}([0, T] ; \boldsymbol{H}) \cap W^{1,2}(0, T ; \boldsymbol{V}) .
$$

\section{An equivalent fixed point problem and exis- tence result}

Let $\boldsymbol{\Phi}:\left(L_{+}^{2}\left(\Xi_{T}\right)\right)^{2} \times\left(L^{2}\left(\Xi_{T}\right)\right)^{3} \rightarrow 2^{\left(L_{+}^{2}\left(\Xi_{T}\right)\right)^{2} \times\left(L^{2}\left(\Xi_{T}\right)\right)^{3}} \backslash\{\emptyset\}$ be the set-valued mapping defined by

$$
\begin{aligned}
& \forall\left(\eta_{1}, \eta_{2}, \boldsymbol{\varsigma}\right) \in\left(L_{+}^{2}\left(\Xi_{T}\right)\right)^{2} \times\left(L^{2}\left(\Xi_{T}\right)\right)^{3} \\
& \boldsymbol{\Phi}\left(\eta_{1}, \eta_{2}, \boldsymbol{\varsigma}\right)=\boldsymbol{\Lambda}^{3}\left(\boldsymbol{u}_{\left(\eta_{1}, \eta_{2}, \boldsymbol{\varsigma}\right)}, \beta_{\left.\boldsymbol{u}_{\left(\eta_{1}, \eta_{2}, \boldsymbol{\varsigma}\right.}\right)}\right)
\end{aligned}
$$

where $\boldsymbol{u}_{\left(\eta_{1}, \eta_{2}, \varsigma\right)}$ is the solution of the variational equation 3.8 which corresponds to $\left(\eta_{1}, \eta_{2}, \boldsymbol{\varsigma}\right)$ according to Lemma 3.2 and $\beta_{\boldsymbol{u}_{\left(\eta_{1}, \eta_{2}, \boldsymbol{\varsigma}\right)}}$ is the solution of (3.6) corresponding to $\boldsymbol{u}=\boldsymbol{u}_{\left(\eta_{1}, \eta_{2}, \boldsymbol{\varsigma}\right)}$.

Since $\left(\lambda_{1}, \lambda_{2}, \gamma\right)$ is a fixed point of $\boldsymbol{\Phi}$, i.e. $\left(\lambda_{1}, \lambda_{2}, \gamma\right) \in \boldsymbol{\Phi}\left(\lambda_{1}, \lambda_{2}, \gamma\right)$, if and only if $\left(\boldsymbol{u}_{\left(\lambda_{1}, \lambda_{2}, \gamma\right)}, \lambda_{1}, \lambda_{2}, \boldsymbol{\gamma}, \beta_{\boldsymbol{u}_{\left(\lambda_{1}, \lambda_{2}, \gamma\right)}}\right)$ is a solution of the Problem $P_{v}^{2}$, we consider the problem which consists in finding a fixed point of the set-valued mapping $\boldsymbol{\Phi}$, called also multivalued function or multifunction.

The existence of a fixed point of the multifunction $\Phi$ will be proved by using a corollary of the Ky Fan's fixed point theorem [17, proved in [30] in the particular case of a reflexive Banach space. We recall this result for the reader's convenience.

Definition 4.1. Let $Y$ be a reflexive Banach space, $D$ a weakly closed set in $Y$, and $F: D \rightarrow 2^{Y} \backslash\{\emptyset\}$ be a set-valued mapping. $F$ is called sequentially weakly upper semicontinuous if $z_{n} \rightarrow z, y_{n} \in F\left(z_{n}\right)$ and $y_{n} \rightarrow y$ imply $y \in F(z)$.

Proposition 4.1. ([30]) Let $Y$ be a reflexive Banach space, $D$ a convex, closed and bounded set in $Y$, and $F: D \rightarrow 2^{D} \backslash\{\emptyset\}$ a sequentially weakly upper semicontinuous set-valued mapping such that $F(z)$ is convex for every $z \in D$. Then $F$ has a fixed point.

Theorem 4.2. Under the assumptions of Sections 2 and 3, there exists $\left(\lambda_{1}, \lambda_{2}, \gamma\right) \in\left(L_{+}^{2}\left(\Xi_{T}\right)\right)^{2} \times\left(L^{2}\left(\Xi_{T}\right)\right)^{3}$ such that $\left(\lambda_{1}, \lambda_{2}, \gamma\right) \in \mathbf{\Phi}\left(\lambda_{1}, \lambda_{2}, \gamma\right)$. For 
each fixed point $\left(\lambda_{1}, \lambda_{2}, \gamma\right)$ of the multifunction $\boldsymbol{\Phi},\left(\boldsymbol{u}_{\left(\lambda_{1}, \lambda_{2}, \gamma\right)}, \lambda, \gamma, \beta_{\left.\boldsymbol{u}_{\left(\lambda_{1}, \lambda_{2}, \gamma\right)}\right)}\right)$, where $\lambda=\lambda_{1}-\lambda_{2}$, is a solution of the Problem $P_{v}^{1}$ and $\left(\boldsymbol{u}_{\left(\lambda_{1}, \lambda_{2}, \gamma\right)}, \lambda_{1}, \lambda_{2}, \gamma, \beta_{\boldsymbol{u}_{\left(\lambda_{1}, \lambda_{2}, \gamma\right)}}\right)$ is a solution of the Problem $P_{v}^{2}$.

Proof. We apply Proposition 4.1 to $Y=\left(L^{2}\left(\Xi_{T}\right)\right)^{5}, F=\boldsymbol{\Phi}$ and $D=$ $\left[\left(L_{+}^{2}\left(\Xi_{T}\right)\right)^{2} \cap D_{0}\right] \times\left[\left(L^{2}\left(\Xi_{T}\right)\right)^{3} \cap D_{1}\right]$.

The set $D \subset\left(L^{2}\left(\Xi_{T}\right)\right)^{5}$ is clearly convex, closed, and bounded.

For all $\boldsymbol{w} \in W^{1,2}(0, T ; \boldsymbol{V}), v \in L^{2}\left(\Xi_{T}\right)$, the set $\boldsymbol{\Lambda}^{3}(\boldsymbol{w}, v)$ is nonempty, closed, and convex, so that $\boldsymbol{\Phi}\left(\eta_{1}, \eta_{2}, \boldsymbol{\varsigma}\right)$ is a nonempty, closed, and convex subset of $D$ for every $\left(\eta_{1}, \eta_{2}, \boldsymbol{\varsigma}\right) \in D$.

In order to prove that the multifunction $\Phi$ is sequentially weakly upper semicontinuous, let $\left(\eta_{1}^{n}, \eta_{2}^{n}, \boldsymbol{\varsigma}^{n}\right) \in D,\left(\delta_{1}^{n}, \delta_{2}^{n}, \varpi^{n}\right) \in \Phi\left(\eta_{1}^{n}, \eta_{2}^{n}, \boldsymbol{\varsigma}^{n}\right) \forall n \in \mathbb{N}$, $\left(\eta_{1}^{n}, \eta_{2}^{n}, \boldsymbol{\varsigma}^{n}\right) \rightarrow\left(\eta_{1}, \eta_{2}, \boldsymbol{\varsigma}\right),\left(\delta_{1}^{n}, \delta_{2}^{n}, \varpi^{n}\right) \rightarrow\left(\delta_{1}, \delta_{2}, \varpi\right)$ and let us verify that $\left(\delta_{1}, \delta_{2}, \varpi\right) \in \Phi\left(\eta_{1}, \eta_{2}, \boldsymbol{\varsigma}\right)$.

We adopt the following notations:

$$
\begin{aligned}
& \boldsymbol{u}_{n}:=\boldsymbol{u}_{\left(\eta_{1}^{n}, \eta_{2}^{n}, \varsigma^{n}\right)}, u_{n N}:=u_{\left(\eta_{1}^{n}, \eta_{2}^{n}, \varsigma^{n}\right) N}, \\
& \boldsymbol{u}_{\eta}:=\boldsymbol{u}_{\left(\eta_{1}, \eta_{2}, \boldsymbol{\varsigma}\right)}, u_{\eta N}:=u_{\left(\eta_{1}, \eta_{2}, \boldsymbol{\varsigma}\right) N}, \\
& \beta_{n}:=\beta_{\boldsymbol{u}_{\left(\eta_{1}^{n}, \eta_{2}^{n}, \varsigma^{n}\right)},}, \beta_{\eta}:=\beta_{\boldsymbol{u}_{\left(\eta_{1}, \eta_{2}, \varsigma\right)}},
\end{aligned}
$$

where $\beta_{\boldsymbol{u}_{\left(\eta_{1}^{n}, \eta_{2}^{n}, \varsigma^{n}\right)}}$ is the solution of 3.6 corresponding to $\boldsymbol{u}_{\left(\eta_{1}^{n}, \eta_{2}^{n}, \varsigma^{n}\right)}$ and $\beta_{\left.\boldsymbol{u}_{\left(\eta_{1}, \eta_{2}, \boldsymbol{s}\right.}\right)}$ is the solution of 3.6 corresponding to $\boldsymbol{u}_{\left(\eta_{1}, \eta_{2}, \boldsymbol{\varsigma}\right)}$.

By Lemma 3.4, we have

$$
\boldsymbol{u}_{n} \rightarrow \boldsymbol{u}_{\eta} \text { in } C^{1}([0, T] ; \boldsymbol{H}) \cap W^{1,2}(0, T ; \boldsymbol{V}),
$$

which implies

$$
\boldsymbol{u}_{n} \rightarrow \boldsymbol{u}_{\eta}, \dot{\boldsymbol{u}}_{n} \rightarrow \dot{\boldsymbol{u}}_{\eta} \quad \text { in }\left(L^{2}\left(\Xi_{T}\right)\right)^{3}
$$

and, by (3.7),

$$
\beta_{n}(t) \rightarrow \beta_{\eta}(t) \text { in } L^{2}(\Xi) \text { for all } t \in[0, T] .
$$

Now, by Lemma 3.1. if $\left(\delta_{1}^{n}, \delta_{2}^{n}, \varpi^{n}\right) \in \Phi\left(\eta_{1}^{n}, \eta_{2}^{n}, \boldsymbol{\varsigma}^{n}\right)=\boldsymbol{\Lambda}^{3}\left(\boldsymbol{u}_{\left(\eta_{1}^{n}, \eta_{2}^{n}, \boldsymbol{\varsigma}^{n}\right)}, \beta_{\left.\boldsymbol{u}_{\left(\eta_{1}^{n}, \eta_{2}^{n}, \varsigma^{n}\right)}\right)}\right)$ for all $n \in \mathbb{N}$, then

$$
\begin{aligned}
& \underline{\kappa}\left(\left[u_{n N}\right], \beta_{n}\right) \leq \delta_{1}^{n}-\delta_{2}^{n} \leq \bar{\kappa}\left(\left[u_{n N}\right], \beta_{n}\right) \text { a.e. in } \Xi_{T}, \\
& \left|\varpi^{n}\right| \leq \mu\left(\dot{\boldsymbol{u}}_{n T}\right)\left(\delta_{1}^{n}+\delta_{2}^{n}\right) \text { a.e. in } \Xi_{T}, \\
& \varpi^{n} \cdot \dot{\boldsymbol{u}}_{n T}+\mu\left(\dot{\boldsymbol{u}}_{n T}\right)\left(\delta_{1}^{n}+\delta_{2}^{n}\right)\left|\dot{\boldsymbol{u}}_{n T}\right|=0 \text { a.e. in } \Xi_{T},
\end{aligned}
$$

for all $n \in \mathbb{N}$. 
First, the relations 4.5 are equivalent to

$$
\int_{\omega} \underline{\kappa}\left(\left[u_{n N}\right], \beta_{n}\right) \leq \int_{\omega}\left(\delta_{1}^{n}-\delta_{2}^{n}\right) \leq \int_{\omega} \bar{\kappa}\left(\left[u_{n N}\right], \beta_{n}\right),
$$

for every measurable subset $\omega \subset \Xi_{T}$ and for all $n \in \mathbb{N}$.

By (4.3) and a converse of Lebesgue's dominated convergence theorem, it follows that there exists a subsequence of $\left(\boldsymbol{u}_{n}\right)_{n}$, denoted by $\left(\boldsymbol{u}_{n_{k}}\right)_{k}$, such that

$$
\left[u_{n_{k} N}\right] \rightarrow\left[u_{\eta N}\right], \dot{\boldsymbol{u}}_{n_{k} T} \rightarrow \dot{\boldsymbol{u}}_{\eta T} \text { a.e. in } \Xi_{T} .
$$

The first convergence property in (4.9) enables to pass to limits in (4.8) with respect to $n_{k}$ according to Fatou's lemma, by using (4.4), the semi-continuity of $\underline{\kappa}$ and $\bar{\kappa}$, the relation (2.2), and the convergence property

$$
\int_{\omega}\left(\delta_{1}^{n}-\delta_{2}^{n}\right) \rightarrow \int_{\omega}\left(\delta_{1}-\delta_{2}\right)
$$

Thus, we obtain

$$
\int_{\omega} \underline{\kappa}\left(\left[u_{\eta N}\right], \beta_{\eta}\right) \leq \int_{\omega}\left(\delta_{1}-\delta_{2}\right) \leq \int_{\omega} \bar{\kappa}\left(\left[u_{\eta N}\right], \beta_{\eta}\right),
$$

for every measurable subset $\omega \subset \Xi_{T}$, which implies

$$
\underline{\kappa}\left(\left[u_{\eta N}\right], \beta_{\eta}\right) \leq \delta_{1}-\delta_{2} \leq \bar{\kappa}\left(\left[u_{\eta N}\right], \beta_{\eta}\right) \text { a.e. in } \Xi_{T} .
$$

Second, the relation 4.6 is equivalent to

$$
\int_{\omega}\left|\varpi^{n}\right| \leq \int_{\omega} \mu\left(\dot{u}_{n T}\right)\left(\delta_{1}^{n}+\delta_{2}^{n}\right)
$$

for every measurable subset $\omega \subset \Xi_{T}$ and for all $n \in \mathbb{N}$. As $\mu(\xi, \cdot)$ is Lipschitz continuous with the Lipschitz constant independent of $\xi$, by using the second convergence property in 4.9 and passing to limits with respect to $n_{k}$ we obtain

$$
\begin{aligned}
\int_{\omega}|\varpi| & \leq \liminf \int_{\omega}\left|\varpi^{n_{k}}\right| \leq \lim \int_{\omega} \mu\left(\dot{\boldsymbol{u}}_{n_{k} T}\right)\left(\delta_{1}^{n_{k}}+\delta_{2}^{n_{k}}\right) \\
& =\int_{\omega} \mu\left(\dot{\boldsymbol{u}}_{\eta T}\right)\left(\delta_{1}+\delta_{2}\right) .
\end{aligned}
$$

Thus

$$
\int_{\omega}|\varpi| \leq \int_{\omega} \mu\left(\dot{\boldsymbol{u}}_{\eta T}\right)\left(\delta_{1}+\delta_{2}\right)
$$


for every measurable subset $\omega \subset \Xi_{T}$, which implies

$$
|\varpi| \leq \mu\left(\dot{\boldsymbol{u}}_{\eta T}\right)\left(\delta_{1}+\delta_{2}\right) \text { a.e. in } \Xi_{T} .
$$

Finally, consider the relation (4.7) which is equivalent to

$$
\int_{\omega} \varpi^{n} \cdot \dot{\boldsymbol{u}}_{n T}+\int_{\omega} \mu\left(\dot{\boldsymbol{u}}_{n T}\right)\left(\delta_{1}^{n}+\delta_{2}^{n}\right)\left|\dot{\boldsymbol{u}}_{n T}\right|=0,
$$

for every measurable subset $\omega \subset \Xi_{T}$ and for all $n \in \mathbb{N}$. By the second convergence property in 4.9 , we have

$$
\mu\left(\dot{\boldsymbol{u}}_{n_{k} T}\right)\left|\dot{\boldsymbol{u}}_{n_{k} T}\right| \rightarrow \mu\left(\dot{\boldsymbol{u}}_{\eta T}\right)\left|\dot{\boldsymbol{u}}_{\eta T}\right| \text { in } L^{1}\left(\Xi_{T}\right),
$$

and, by the relations 2.2 , 4.5,

$$
\delta_{1}^{n}+\delta_{2}^{n} \rightarrow^{*} \delta_{1}+\delta_{2} \text { in } L^{\infty}\left(\Xi_{T}\right) .
$$

Passing to limits in 4.12 by using 4.13 and 4.14, we obtain

$$
\int_{\omega} \varpi \cdot \dot{\boldsymbol{u}}_{\eta T}+\int_{\omega} \mu\left(\dot{\boldsymbol{u}}_{\eta T}\right)\left(\delta_{1}+\delta_{2}\right)\left|\dot{\boldsymbol{u}}_{\eta T}\right|=0,
$$

for every measurable subset $\omega \subset \Xi_{T}$, which implies

$$
\varpi \cdot \dot{\boldsymbol{u}}_{\eta T}+\mu\left(\dot{\boldsymbol{u}}_{\eta T}\right)\left(\delta_{1}+\delta_{2}\right)\left|\dot{\boldsymbol{u}}_{\eta T}\right|=0 .
$$

From 4.10, 4.11, 4.15, it follows that $\left(\delta_{1}, \delta_{2}, \varpi\right) \in \Phi\left(\eta_{1}, \eta_{2}, \varsigma\right)$, so that we may apply the Proposition 4.1 to conclude the proof.

\section{References}

[1] L.E. Andersson, Existence results for quasistatic contact problems with Coulomb friction, Appl. Math. Optim. 42 (2000) 169-202.

[2] V. Barbu, Nonlinear Differential Equations of Monotone Types in Banach Spaces, Springer, 2010.

[3] P. Boieri, F. Gastaldi, D. Kinderlehrer, Existence, uniqueness, and regularity results for the two-body contact problem, Appl. Math. Optim. 15 (1987) 251-277.

[4] O. Chau, W. Han, M. Sofonea, A dynamic frictional contact problem with normal damped response, Acta Applicandae Mathematicae 71 (2002) $159-178$. 
[5] O. Chau, M. Shillor, M. Sofonea, Dynamic frictionless contact with adhesion, Z. Angew. Math. Phys. 55 (2004) 32-47.

[6] M. Cocou, Existence of solutions of a dynamic Signorini's problem with nonlocal friction in viscoelasticity, Z. Angew. Math. Phys. 53 (2002) 10991109.

[7] M. Cocou, A class of implicit evolution inequalities and applications to dynamic contact problems, Annals of the University of Bucharest (mathematical series) 4 (LXII) (2013) 167-178.

[8] M. Cocou, A variational analysis of a class of dynamic problems with slip dependent friction, Annals of the University of Bucharest (mathematical series) 5 (LXIII) (2014) 279-297.

[9] M. Cocou, A class of dynamic contact problems with Coulomb friction in viscoelasticity, Nonlinear Analysis: RWA 22 (2015) 508-519.

[10] M. Cocou, A variational inequality and applications to quasistatic problems with Coulomb friction, Applicable Analysis 97 (2018) 1357-1371.

[11] M. Cocou, A dynamic viscoelastic problem with friction and ratedepending contact interactions, Evolution Equations and Control Theory 9 (2020) 981-993.

[12] M. Cocou, R. Rocca, Existence results for unilateral quasistatic contact problems with friction and adhesion, Math. Modelling and Num. Analysis 4 (2000) 981-1001.

[13] M. Cocou, G. Scarella, Analysis of a dynamic unilateral contact problem for a cracked viscoelastic body, Z. Angew. Math. Phys. 57 (2006) 523-546.

[14] M. Cocou, M. Schryve, M. Raous, A dynamic unilateral contact problem with adhesion and friction in viscoelasticity, Z. Angew. Math. Phys. 61 (2010) 721-743.

[15] N. Cristescu, I. Suliciu, Viscoplasticity, Martinus Nijhoff Publishers, Editura Technica, 1982.

[16] C. Eck, J. Jarušek, M. Krbec, Unilateral Contact Problems - Variational Methods and Existence Theorems, Chapman \& Hall/CRC, 2005.

[17] K. Fan, Fixed points and minimax theorems in locally convex topological linear spaces, Proc. Nat. Acad. Sci. USA 38 (1952) 121-126. 
[18] M. Frémond, Adhérence des solides, Journal de Mécanique Théorique et Appliquée 6 (1987) 383-407.

[19] M. Frémond, Contact with adhesion, in J.J. Moreau and P.D. Panagiotopoulos (Eds.), Nonsmooth Mechanics and Applications, CISM Courses and Lectures No. 302, Springer-Verlag, 1988, pp. 177-221.

[20] K.L. Kuttler, Dynamic friction contact problems for general normal and friction laws, Nonlinear Analysis: TMA 28 (1997) 559-575.

[21] K.L. Kuttler, M. Shillor, Dynamic bilateral contact with discontinuous friction coefficient, Nonlinear Analysis: TMA 45 (2001) 309-327.

[22] K.L. Kuttler, M. Shillor, Dynamic contact with Signorini's condition and slip rate depending friction, Electronic J. Differential Equations 83 (2004) 1-21.

[23] K.L. Kuttler, M. Shillor, J.R. Fernández, Existence and regularity for dynamic viscoelastic adhesive contact with damage, Appl. Math. Optim. 53 (2006) 31-66.

[24] J.A.C. Martins, J.T. Oden, Existence and uniqueness results for dynamic contact problems with nonlinear normal and friction interface laws, Nonlinear Analysis: TMA 11 (1987) 407-428.

[25] J.C. Mergel, R. Sahli, J. Scheibert, R.A. Sauer, Continuum contact models for coupled adhesion and friction, The Journal of Adhesion 95 (2019) $1101-1133$.

[26] S. Migorski, A. Ochal, A unified approach to dynamic contact problems in viscoelasticity, J. Elasticity 83 (2006) 247-275.

[27] S. Migórski, A. Ochal, M. Sofonea, Nonlinear Inclusions and Hemivariational Inequalities, Springer, 2013.

[28] Z. Naniewicz, P.D. Panagiotopoulos, Mathematical Theory of Hemivariational Inequalities and Applications, Marcel Dekker, Inc., 1995.

[29] P.D. Panagiotopoulos, Hemivariational Inequalities: Applications in Mechanics and Engineering, Springer, 1993.

[30] P.J. Rabier, O.V. Savin, Fixed points of multi-valued maps and static Coulomb friction problems, J. Elasticity 58 (2000) 155-176. 
[31] M. Raous, L. Cangémi, M. Cocou, A consistent model coupling adhesion, friction, and unilateral contact, Comput. Meth. Appl. Mech. Engrg. 177 (1999) 383-399.

[32] R. Rocca, M. Cocou, Existence and approximation of a solution to quasistatic Signorini problem with local friction, Int. J. Engrg. Sci. 39 (2001) $1233-1255$.

[33] R. Rocca, M. Cocou, Numerical analysis of quasistatic unilateral contact problems with local friction, SIAM Journal on Numerical Analysis 39 (2001) 1324-1342.

[34] M. Shillor, M. Sofonea, J.J. Telega, Models and Analysis of Quasistatic Contact, Lect. Notes Phys. 655, Springer, 2004.

[35] J. Simon, Compact sets in the space $L^{p}(0, T ; B)$, Ann. Mat. Pura Appl. 146 (1987) 65-96.

[36] M. Sofonea, W. Han, M. Shillor, Analysis and Approximation of Contact Problems with Adhesion or Damage, Chapman \& Hall/CRC, 2006. 